\title{
Dietary inflammatory index (DII) may be associated with hypertriglyceridemia waist circumference phenotype in overweight and obese Iranian women: a cross sectional study
}

Atefeh Tavakoli ${ }^{1}$, Atieh Mirzababaei ${ }^{1}$, Hanieh Moosavi ${ }^{1}$, Sanaz Mehranfar ${ }^{1}$, Seyed-Ali Keshavarz ${ }^{2}$ and Khadijeh Mirzaei ${ }^{1 *}$ (1)

\begin{abstract}
Objective: Recent studies have shown that increased dietary inflammatory index (DII) score or consumption of pro-inflammatory foods can lead to increased waist circumference (WC) as well as triglyceride (TG) concentrations in obese people. The purpose of this study is to examine the association between DII and hypertriglyceridemic waist circumference phenotype (HTGWCP) in women with overweight and obesity.

Results: There was a positive significant correlation between DII and HTGWCPs. In other words, with an increase in DII score or higher consumption of pro-inflammatory foods, the odds of having abnormal phenotypes including; enlarged waist normal TG (EWNT) $(\mathrm{OR}=2.85,95 \% \mathrm{Cl} 1.02$ to 7.98 , P for trend $=0.04)$, normal waist enlarged TG (NWET) $(\mathrm{OR}=5.85,95 \% \mathrm{Cl} 1.1$ to $31.11, \mathrm{P}$ for trend $=0.03)$ and enlarged waist enlarged TG (EWET) $(\mathrm{OR}=3.13,95 \% \mathrm{Cl} 0.95$ to 10.27, $\mathrm{P}$ for trend $=0.05$ ) increase compared to normal waist normal TG (NWNT) phenotype. In conclusion; increasing DII scores can increase abnormal phenotypes and therefore may increase WC and TG levels in overweight and obese women.
\end{abstract}

Keywords: Dietary inflammatory index, Hypertriglyceridemic waist circumference phenotype, Obesity

\section{Introduction}

Obesity is an important public health issue in all over the world and it is emerging as a threat in more affluent sectors of developing countries [1]. According to the World Health Organization (WHO) in 2008, more than $10 \%$ of the world's adult population, about 500 million people were obese [2]. This issue is associated with chronic

\footnotetext{
*Correspondence: mirzaei_kh@tums.ac.ir

${ }^{1}$ Department of Community Nutrition, School of Nutritional Sciences and Dietetics, Tehran University of Medical Sciences (TUMS), P.O. Box: 14155-6117, Tehran, Iran

Full list of author information is available at the end of the article
}

diseases including; cardiovascular disease (CVD), diabetes, breast and endometrial cancer in women [1]. Obesity is usually caused by the accumulation of excess fat over the body and it is often characterized as a state of lowgrade chronic inflammation [3].

Many studies have shown an association between diet and inflammatory markers and chronic inflammatoryrelated diseases such as obesity [4-6]. Healthy dietary patterns, such as the Mediterranean diet (MeDiet), can reduce the low-grade inflammation observed in obese persons due to some of their components are known as an anti-inflammatory dietary pattern [7-10]. The DII 
as a new tool to assess inflammatory potential of the diet determines the inflammatory potential of the diet on a continuum from maximally anti- to pro-inflammatory [11]. The results of a study showed that DII was directly associated with the incidence of obesity, especially abdominal obesity in women compared to men with higher body mass index (BMI), WC, waist-to-height ratio (WHtR). According to results of this study, DII was inversely related with the consumption of anti-inflammatory nutrients and foods $[12,13]$. A study concluded that unhealthy diets such as the Western diet, which are associated with higher inflammation, increased metabolic syndrome (MetS) components, such as WC and TG levels, compared with the MeDiet [4]. Several investigations resulted that the inflammatory properties of the diet were associated with higher TG and lower high-density lipoprotein (HDL) plasma levels $[14,15]$. The HTGWCP is defined as high serum TG levels and simultaneous presence of high WC and also it is a marker for assessing visceral obesity and low-grade inflammation status such as obesity [16]. Due to the ascending growth of obesity and the strong association of this chronic inflammatory condition with many other chronic diseases [1], as well as the results of many previous studies, especially cohort studies [15, 17], which indicate the relationship between pro-inflammatory diet patterns and metabolic syndrome components, especially WC, HDL and TG, as well as some other anthropometric indices such as fat free mass (FFM), we weighed to examine the association of DII with obesity $[18,19]$. On the other hand, studies have shown that following pro-inflammatory diets raises the levels of many inflammatory markers, including; Tumor necrosis factor alpha (TNF $\alpha$ ), high-sensitivity C-reactive protein (hs-CRP), Interleukin1beta (IL-1 $\beta$ ), Interleukin 6 (IL-6), Interleukin 10 (IL-10), in obese or chronically ill people, especially in women, which in turn has led to the spread of obesity in these people [13, 20,21]. Due to the prevalence of obesity, abdominal obesity and malnutrition in developing societies, especially among women, as well as the limitations of studies conducted on this sensitive group of society [22-24] and also the greater likelihood of the spread of inflammatory conditions, such as viral [25] and respiratory inflammatory diseases [26] in women compared to men and also according to our investigations, the majority of studies on the relationship between DII and obesity and many other diseases are more in women compared to men [27-29]. In this regard, a 20-year cohort study was performed on 70,991 women with diabetes and the effect BMI mediator was also examined in this study, the results of which showed a direct relationship between the incidence of diabetes and DII through BMI [30]. So, we selected women as the target group.
There is no investigation on the association between DII and HTGWCP, we aimed to evaluate the relationship between DII and HTGWCP among obese and overweight women.

\section{Main text \\ Method \\ Study population}

226 women aged 18 to 48 years were participated. The sample size was calculated based on the following formula: $\mathrm{n}=[(\mathrm{Z} 1-\alpha+\mathrm{Z} 1-\beta) \times \sqrt{ } 1-\mathrm{r} 2] / \mathrm{r}) 2+2)$, which $\alpha=0.05, \beta=0.95, r=0.25$, and then, with $80 \%$ power and $95 \%$ confidence. BMI range for women participants was between 25 to $49.6 \mathrm{~kg} / \mathrm{m}^{2}$. Inclusion criteria: aged 18 to 48 years, overweight or obese women (BMI $\geq 25 \mathrm{~kg}$ / $\mathrm{m}^{2}$ ) and exclusion criteria: menopausal pregnancy or lactation period, diabetes mellitus, liver and kidney disorders, hypertension, CVD, smoking and alcohol consumption, chronic diseases affecting the dietary pattern. Participating women signed the consent form. The protocol was accepted by the Ethical Commission at Tehran University of Medical Sciences (IR.TUMS.MEDICINE. REC.1399.636).

\section{Anthropometric assessment}

Weight was measured with the use of a digital scale (Seca, Hamburg, Germany) with the least clothes and without shoes. Height was measured using a calibrated height gauge (in the standing position without shoes). hip circumference $(\mathrm{HC})$ and WC were measured in the largest and the smallest girth, respectively. BMI was calculated as weight $(\mathrm{kg})$ to height $\left(\mathrm{m}^{2}\right)$ ratio. skeletal muscle mass (SMM), FFM, waist hip ratio (WHR) as body composition components; evaluated using a body composition analyzer (InBody770 scanner; InBody, Seoul, Korea).

\section{Biochemical assessment}

After 10 to $12 \mathrm{~h}$ of fasting, blood samples were taken and the serum was stored at $-80{ }^{\circ} \mathrm{C}$. All samples were analyzed by using a single assay according to manufacturer's protocol. Glucose oxidase method was using for fasting plasma glucose assessment and insulin level was measured by using an enzyme-linked immunosorbent assay (ELISA) kit (Human insulin ELISA kit, DRG Pharmaceuticals, GmbH, Germany). TG, Total cholesterol (T-Chole), Low-density lipoprotein (LDL), HDL were measured by related kits (Pars Azemun, Iran). serum glutamic-pyruvic transaminase (SGPT) and serum glutamicoxaloacetic transaminase (SGOT) were assessed using the International Federation of Clinical Chemistry and Laboratory Medicine standardization. The Homeostatic model assessment for insulin resistance (HOMA-IR) was 
estimated as the product of fasting glucose and insulin level divided by 22.5 with molar unit ( $\mathrm{mmol} / \mathrm{L})$.

\section{Demographic variables}

Demographic questionnaire was used for evaluation of qualitative variables such as education, job, marital situation, economic status and familial obesity history.

\section{Dietary inflammatory index}

More negative DII or a lower DII score indicates eat less inflammatory foods and more positive DII or a higher DII score indicates eat more inflammatory foods. Dietary intake was assessed by using a semi-quantitative food frequency questionnaire (FFQ) contains 147 Iranian food points, with a list of foods and their standard serving sizes that assesses the usual food intake in the past year. The validity and reliability of FFQ have been confirmed [31]. Software program Nutritionist IV was used for nutrient analysis which was modified for Iranian foods. DII was computed based on Shivappa et al. and Esfahani et al. methods [32, 33]. To calculate DII, first, the Z-score was computed by subtracting the global standard mean of the declared food amounts and so dividing the difference by the global standard deviation. After that, the Z-score was converted to a centered percentile score and this was multiplied by the food parameter effect score, to obtain a DII score for a subject. To create the final DII score, all of the food parameter-specific DII scores were summed.

\section{Hypertriglyceridemic waist circumference phenotype}

Women were categorized in 4 phenotype groups according to the cutoff points: NWNT: normal WC $<88 \mathrm{~cm}$ and normal serum TG concentrations $<150 \mathrm{mg} / \mathrm{dL}$ ), EWNT: enlarged $W C \geq 88 \mathrm{~cm}$ and normal serum TG concentration $<150 \mathrm{mg} / \mathrm{dL}$ ), NWET: normal $\mathrm{WC}<88 \mathrm{~cm}$ and elevated TG $\geq 150 \mathrm{mg} / \mathrm{dL}$, EWET: enlarged $\mathrm{WC} \geq 88 \mathrm{~cm}$ and hyper serum TG concentration $\geq 150 \mathrm{mg} / \mathrm{dL}$ [34].

\section{Statistical analyzes}

The normal distribution of data was checked by Kolmogorov Smirnov test. Quantitative variables were described using mean and standard deviation and qualitative variables by reporting number and percentage. Analysis of variance (ANOVA) test was confirmed for calculating of relationships between DII and HTGWCP and quantitative variables and also the tukey's post hoc test was using for the adjustment of multiple comparisons. Relationships of DII, HTGWCP and qualitative variables was conducted by Chi-square test. Analysis of covariance (ANCOVA) was used to modify the effect of confounders included: age, energy intake, BMI and physical activity. Associations between DII and HTGWCP were confirmed in Multinomial logistic regression to adjust for confounder points; energy intake, age, insulin plasma level, marital status, education, economic status and family history of obesity. We considered the group of "NWNT" about HTGWCPs as reference. Statistical analyzes were performed using IBM SPSS version 25.0 (SPSS, Chicago, IL, USA). The level of significance was considered as being a $P$ value $\leq 0.05$.

\section{Result}

\section{Study population characteristics}

The mean \pm standard deviation (SD) of age, weight, BMI, WC were $36.67 \pm 9.10$ years, $81.29 \pm 12.43 \mathrm{~kg}$, $31.26 \pm 4.29 \mathrm{~kg} / \mathrm{m}^{2}, \quad 99.61 \pm 10.07 \mathrm{~cm}$, respectively. NWNT, EWNT, NWET, EWET phenotypes included $6.2 \%, 42 \%, 5.9,45 \%$ of individuals respectively. $70.8 \%$ of women were married, $84.7 \%$ of them had a university education and $61.9 \%$ of them were unemployed.

\section{Correlation analysis}

After controlling the confounding variables including age, energy intake, BMI and physical activity: a significant correlation was found between DII and TG, and this variable had a higher mean in the second tertile of DII $(\mathrm{P}=0.02)$ Table 1, Additional file 1: Table S1.

After adjustment for confounders including; age, energy intake, BMI and physical activity through ANCOVA test insulin $(\mathrm{P}=0.01)$ and HOMA-IR, TG, weight, height, BMI, FFM, WHR, WC $(\mathrm{P}<0.001)$ and SMM $(P=0.002)$, were all significant with HTGWPs and TG concentrations, weight, BMI, SMM and FFM were higher in EWET phenotype, insulin and HOMA-IR were higher in NWET phenotype and height, WHR and WC were higher in EWNT phenotype Table 2, Additional file 2: Table S2.

In crude model a significant correlation was showed between DII and EWET (OR=2.07, 95\% CI 0.42 to 0.69 , $\mathrm{P}$ for trend $=0.02$ ). By adjusting variables included; energy intake, age, plasma insulin levels, marital status, education, economic status and familial obesity history, we observed that with increasing DII, the odds of having the EWNT $(\mathrm{OR}=2.85,95 \% \mathrm{CI} 0.77$ to 0.86 , $\mathrm{P}$ for trend $=0.04)$, NWET $(\mathrm{OR}=5.85,95 \% \mathrm{CI} 0.56$ to $0.72, \mathrm{P}$ for trend $=0.03)$ and EWET $(\mathrm{OR}=3.13,95 \% \mathrm{CI} 0.86$ to 0.98 , P for trend $=0.05$ ) phenotypes were significantly increased compared to NWNT phenotype among participants Table 3, Additional file 3: Table S3.

\section{Discussion}

The present investigation was the first cross-sectional study that evaluated the association between DII and HTGWCP among overweight and obese women. We have shown that DII is significantly associated with 
Table 1 Description of characteristics among tertiles of DII

\begin{tabular}{|c|c|c|c|c|c|}
\hline Variables & $\begin{array}{l}\mathrm{T} 1 \\
\mathrm{~N}=76\end{array}$ & $\begin{array}{l}\mathrm{T} 2 \\
\mathrm{~N}=77\end{array}$ & $\begin{array}{l}\mathrm{T} 3 \\
\mathrm{~N}=73\end{array}$ & Pvalue $^{*}$ & P value ${ }^{* *}$ \\
\hline Age (year) & $37.03(8.52)^{a}$ & $37.06(8.22)$ & $34.82(8.43)$ & 0.12 & 0.08 \\
\hline PA (MET h/week) & $1200.87(1376.69)$ & $1173.54(1555.11)$ & $1143.56(2725.58)$ & 0.98 & 0.97 \\
\hline \multicolumn{6}{|l|}{ Blood parameters } \\
\hline FBS (mg/dL) & $85.74(8.06)$ & $88.51(10.31)$ & $87.22(10.50)$ & 0.21 & 0.13 \\
\hline Insulin $(\mu \mid \mathrm{U} / \mathrm{mL})$ & $1.26(0.23)$ & $1.24(0.21)$ & $1.22(0.25)$ & 0.63 & 0.99 \\
\hline HOMA-IR & $3.63(1.62)^{3}$ & $3.19(1.25)$ & $2.87(0.93)^{1}$ & $0.02^{\mathrm{a}}$ & 0.09 \\
\hline $\mathrm{TC}(\mathrm{mg} / \mathrm{dL})$ & $184.02(39.14)$ & $188.66(33.93)$ & $182.42(35.14)$ & 0.54 & 0.42 \\
\hline $\mathrm{HDL}-\mathrm{C}(\mathrm{mg} / \mathrm{dL})$ & $47.37(13.06)$ & $46.01(10.10)$ & $46.77(10.38)$ & 0.75 & 0.74 \\
\hline LDL-C (mg/dL) & $95.12(26.36)$ & $97.12(23.05)$ & 91.69 (21.95) & 0.36 & 0.25 \\
\hline $\mathrm{TG}(\mathrm{mg} / \mathrm{dL})$ & $145.91(68.73)$ & $108.09(43.03)$ & $108.84(61.55)^{1,2}$ & $0.002^{b}$ & 0.01 \\
\hline SGOT (mg/dL) & $17.00(5.99)$ & 18.54 (9.19) & $17.93(6.68)$ & 0.44 & 0.42 \\
\hline SGPT (mg/dL) & $17.60(9.72)$ & $20.20(16.12)$ & $19.42(11.63)$ & 0.44 & 0.49 \\
\hline \multicolumn{6}{|c|}{ Body composition parameters } \\
\hline $\mathrm{BMI}\left(\mathrm{kg} / \mathrm{m}^{2}\right)$ & $31.09(3.88)$ & $30.37(4.65)$ & $31.25(4.10)$ & 0.33 & 0.11 \\
\hline SMM (kg) & $25.67(2.98)$ & $25.54(3.29)$ & $25.66(3.82)$ & 0.96 & 0.95 \\
\hline FFM (kg) & $46.74(5.02)$ & $46.60(5.58)$ & $46.72(6.49)$ & 0.98 & 0.94 \\
\hline WHR & 1.94 (9.59) & $0.93(0.05)$ & $0.93(0.05)$ & 0.37 & 0.85 \\
\hline$W C(\mathrm{~cm})$ & $98.97(9.45)$ & $97.76(10.19)$ & $99.52(10.18)$ & 0.48 & 0.38 \\
\hline \multicolumn{6}{|l|}{ Qualitative variables } \\
\hline \multicolumn{6}{|l|}{ Economic status } \\
\hline Poor & $12(41.4 \%)^{b}$ & $7(20.7 \%)$ & $11(37.9 \%)$ & $0.67^{* * *}$ & 0.77 \\
\hline Moderate & $35(34.8 \%)$ & 33 (33.9\%) & $32(31.3 \%)$ & & \\
\hline Good & $29(32.8 \%)$ & $32(34.4 \%)$ & $29(32.8 \%)$ & & \\
\hline Excellent & $2(12.5 \%)$ & $5(50 \%)$ & $3(37.5 \%)$ & & \\
\hline \multicolumn{6}{|l|}{ Education status } \\
\hline Illiterate & 1 (33.3\%) & $1(33.3 \%)$ & $1(33.3 \%)$ & 0.46 & 0.37 \\
\hline Diploma & $11(38.9 \%)$ & $7(19.4 \%)$ & $12(41.7 \%)$ & & \\
\hline University educated & $64(33 \%)$ & $69(35.3 \%)$ & $60(31.7 \%)$ & & \\
\hline \multicolumn{6}{|l|}{ Marriage status } \\
\hline Single & $14(26.8 \%)$ & $20(39.3 \%)$ & $19(33.9 \%)$ & 0.38 & 0.17 \\
\hline Married & $62(35.7 \%)$ & $57(31.4 \%)$ & $63(32.9 \%)$ & & \\
\hline \multicolumn{6}{|l|}{ Familial obesity history } \\
\hline Yes & $55(34.2 \%)$ & $56(34.7 \%)$ & $50(31 / 1 \%)$ & 0.24 & 0.16 \\
\hline No & $21(31.1 \%)$ & $18(27.3 \%)$ & $23(41.6 \%)$ & & \\
\hline \multicolumn{6}{|c|}{ Hypertriglyceridemic waist circumference phenotype } \\
\hline NWNT & $3(16.7 \%)$ & $5(27.8 \%)$ & $8(55.5 \%)$ & 0.26 & 0.19 \\
\hline EWNT & $21(29.2 \%)$ & $32(39.3 \%)$ & $25(31.5 \%)$ & & \\
\hline NWET & 7 (30.4\%) & $8(34.8 \%)$ & $8(34.8 \%)$ & & \\
\hline EWET & $45(38.6 \%)$ & $32(30.0 \%)$ & $32(31.4 \%)$ & & \\
\hline
\end{tabular}

Bold values indicate the significant range as a tabular foot

$\mathrm{T} 1, \mathrm{~T} 2$ and $\mathrm{T} 3$ are $\mathrm{DII}$ tertiles

DIl dietary inflammatory index, PA physical activity, FBS Fasting blood sugar, HOMA-IR Homeostatic Model Assessment for Insulin Resistance, TC total cholesterol, HDL-C high density lipoprotein cholesterol, LDL-C low density lipoprotein cholesterol, TG triglyceride, SGOT serum glutamic-oxaloacetic transaminase, SGPT Serum glutamic-pyruvic transaminase, BMI: body mass index, SMM Skeletal muscle mass, FFM fat free mass, WHR waist to hip ratio, WC waist circumference, NWNT normal waist normal triglyceride, EWNT enlarged waist normal triglyceride, NWET normal waist enlarged triglyceride, EWET enlarged waist enlarged triglyceride

* P value resulted from ANOVA analysis

** P value reported after adjusting age, energy intake, BMI and physical activity with ANCOVA

*** P value resulted from chi-squared test analysis

${ }^{\text {a }}$ Mean $\pm S D$

${ }^{\mathrm{b}} \mathrm{N}(\%), \mathrm{P}$ value $\leq 0.05$ is significant 
Table 2 Description of characteristics among types of hypertrigeliceridemic waste phenotype

\begin{tabular}{|c|c|c|c|c|c|c|}
\hline \multirow[t]{2}{*}{ Variables } & \multicolumn{4}{|c|}{ Hypertriglyceridemic waist circumference phenotypes } & \multirow[t]{2}{*}{ Pvalue ${ }^{*}$} & \multirow[t]{2}{*}{ Pvalue ${ }^{* *}$} \\
\hline & $\begin{array}{l}\text { NWNT } \\
\mathrm{N}=15\end{array}$ & $\begin{array}{l}\text { EWNT } \\
N=88\end{array}$ & $\begin{array}{l}\text { NWET } \\
N=14\end{array}$ & $\begin{array}{l}\text { EWET } \\
N=109\end{array}$ & & \\
\hline Age (year) & $32.08 \pm 8.08^{\mathrm{a}, 4}$ & $36.87 \pm 9.78$ & $36.20 \pm 7.18$ & $37.17 \pm 8.66^{1}$ & 0.06 & 0.17 \\
\hline PA (MET h/week) & $682 \pm 832.81$ & $973.86 \pm 1158.23$ & $1972 \pm 4117.85$ & $1277.48 \pm 2171$ & 0.29 & 0.22 \\
\hline \multicolumn{7}{|l|}{ Blood parameters } \\
\hline Insulin $(\mu \mathrm{I} \mathrm{U} / \mathrm{mL})$ & $1.21 \pm 0.18$ & $1.19 \pm 0.23^{4}$ & $1.37 \pm 0.18$ & $1.29 \pm 0.24^{2}$ & 0.01 & 0.01 \\
\hline HOMA-IR & $3.32 \pm 1.35^{4}$ & $3.09 \pm 1.03^{3,4}$ & $4.72 \pm 1.32^{2}$ & $4.14 \pm 1.56^{1,2}$ & $<0.001$ & $<0.001$ \\
\hline $\mathrm{TC}(\mathrm{mg} / \mathrm{dL})$ & $168.81 \pm 26.56$ & $188.05 \pm 38.15$ & $173.37 \pm 29.49$ & $187.70 \pm 35.52$ & 0.06 & 0.73 \\
\hline $\mathrm{HDL}-\mathrm{C}(\mathrm{mg} / \mathrm{dL})$ & $49.56 \pm 10.71$ & $45.75 \pm 11.16$ & $46.66 \pm 11.51$ & $46.74 \pm 10.60$ & 0.63 & 0.97 \\
\hline LDL-C (mg/dL) & $86.68 \pm 18.82$ & $94.70 \pm 26.51$ & $93.41 \pm 20.33$ & $97.10 \pm 23.65$ & 0.40 & 0.76 \\
\hline $\mathrm{TG}(\mathrm{mg} / \mathrm{dL})$ & $93.32 \pm 27.13^{3,4}$ & $92.34 \pm 27.79^{3,4}$ & $189.00 \pm 30.41^{1,2}$ & $213.52 \pm 49.52^{1,2}$ & $<0.001$ & $<0.001$ \\
\hline $\mathrm{SGOT}(\mathrm{mg} / \mathrm{dL})$ & $17.62 \pm 5.48$ & $18.11 \pm 7.72$ & $16.29 \pm 5.05$ & $18.40 \pm 8.41$ & 0.67 & 0.63 \\
\hline $\mathrm{SGPT}(\mathrm{mg} / \mathrm{dL})$ & $13.93 \pm 6.24$ & $20.22 \pm 13.92$ & $17.37 \pm 8.01$ & $20.09 \pm 15.13$ & 0.30 & 0.62 \\
\hline \multicolumn{7}{|c|}{ Body composition parameters } \\
\hline Weight(cm) & $65.88 \pm 3.45^{2,4}$ & $83.06 \pm 11.68^{1,3}$ & $65.44 \pm 3.01^{2,4}$ & $83.85 \pm 11.64^{1,3}$ & $<0.001$ & $<0.001$ \\
\hline Height(cm) & $159.26 \pm 4.84$ & $161.78 \pm 5.79^{3}$ & $156.70 \pm 4.79^{2,4}$ & $161.55 \pm 5.96^{3}$ & $<0.001$ & $<0.001$ \\
\hline BMI $\left(\mathrm{kg} / \mathrm{m}^{2}\right)$ & $25.96 \pm 0.86^{2,4}$ & $31.76 \pm 4.13^{1,3}$ & $26.72 \pm 1.26^{2,4}$ & $32.11 \pm 4.12^{1,3}$ & $<0.001$ & $<0.001$ \\
\hline $\mathrm{SMM}(\mathrm{kg})$ & $22.08 \pm 2.19^{2,4}$ & $25.75 \pm 3.14^{1,3}$ & $22.48 \pm 1.95^{2,4}$ & $26.26 \pm 3.52^{1,3}$ & $<0.001$ & 0.002 \\
\hline FFM (kg) & $40.81 \pm 3.66^{2,4}$ & $46.79 \pm 5.09^{1,3}$ & $41.44 \pm 3.39^{2,4}$ & $47.73 \pm 5.93^{1,3}$ & $<0.001$ & $<0.001$ \\
\hline WHR & $0.86 \pm 0.18$ & $1.48 \pm 6.98$ & $0.86 \pm 0.02$ & $0.94 \pm 0.04$ & 0.68 & $<0.001$ \\
\hline$W C(\mathrm{~cm})$ & $84.66 \pm 2.16^{2,3,4}$ & $101.74 \pm 8.99^{1,3}$ & $85.02 \pm 1.85^{2,4}$ & $101.60 \pm 8.97^{1,3}$ & $<0.001$ & $<0.001$ \\
\hline \multicolumn{7}{|l|}{ Qualitative variables } \\
\hline \multicolumn{7}{|l|}{ Economic status } \\
\hline Poor & $2(5.0 \%)^{b}$ & $13(32.5 \%)$ & $2(5.0 \%)$ & $20(57.5 \%)$ & $0.18^{* * *}$ & 0.65 \\
\hline Moderate & $7(7.8 \%)$ & 33 (41.9\%) & $6(6.6 \%)$ & 47 (43.7\%) & & \\
\hline Good & $5(5.8 \%)$ & $27(41.3 \%)$ & $6(7.1 \%)$ & $38(45.8 \%)$ & & \\
\hline Excellent & $1(5.0 \%)$ & $15(75.1 \%)$ & $0(0 \%)$ & $4(19.9 \%)$ & & \\
\hline \multicolumn{7}{|l|}{ Education status } \\
\hline Illiterate & $0(0.0 \%)$ & $1(25.0 \%)$ & $1(25.0 \%)$ & $4(50.0 \%)$ & 0.31 & 0.88 \\
\hline Diploma & $1(2.0 \%)$ & $17(36.7 \%)$ & $2(4.1 \%)$ & $31(57.2 \%)$ & & \\
\hline University educated & $14(7.0 \%)$ & $70(43.3 \%)$ & $11(6.1 \%)$ & $74(43.6 \%)$ & & \\
\hline \multicolumn{7}{|l|}{ Marital status } \\
\hline Single & $5(7.3 \%)$ & $36(52.3 \%)$ & $3(3.7 \%)$ & $29(36.7 \%)$ & 0.05 & 0.47 \\
\hline Married & $10(5.9 \%)$ & $52(38.5 \%)$ & $11(7.0 \%)$ & $80(48.6 \%)$ & & \\
\hline \multicolumn{7}{|l|}{ Familial obesity history } \\
\hline Yes & $7(5.2 \%)$ & $56(39.7 \%)$ & $9(6.7 \%)$ & $85(48.4 \%)$ & 0.17 & 0.92 \\
\hline No & $8(9.3 \%)$ & $32(47.2 \%)$ & $5(5.5 \%)$ & $24(38 \%)$ & & \\
\hline
\end{tabular}

Bold values indicate the significant range as a tabular foot

NWNT normal waist normal triglyceride, EWNT enlarged waist normal triglyceride, NWET normal waist enlarged triglyceride, EWET enlarged waist enlarged triglyceride, $P A$ physical activity, HOMA-IR Homeostatic Model Assessment for Insulin Resistance, $T C$ total cholesterol, HDL-C high density lipoprotein cholesterol, $L D L-C$ low density lipoprotein cholesterol, TG triglyceride, SGOT serum glutamic-oxaloacetic transaminase, SGPT Serum glutamic-pyruvic transaminase, BMI body mass index, SMM skeletal muscle mass, FFM fat free mass, WHR waist to hip ratio, WC waist circumference

* P value resulted from ANOVA analysis

** P value reported after adjusting age, energy intake, BMI and physical activity with ANCOVA analysis

*** $\mathrm{P}$ value resulted from chi-squared test analysis

${ }^{\text {a }}$ Mean \pm SD

${ }^{\mathrm{b}} \mathrm{N}(\%), \mathrm{P}$ value $\leq 0.05$ is significant 
Table 3 Crude model and adjusted model for Relationship between hyper triglyceridemic waist circumference phenotype and DII

\begin{tabular}{|c|c|c|c|}
\hline & OR $(95 \% \mathrm{Cl})$ & $\beta \pm S E$ & P-trend \\
\hline \multicolumn{4}{|c|}{ Crude model } \\
\hline EWNT & 1.80 (0.91 to1.01) & $0.58 \pm 0.34$ & 0.08 \\
\hline NWET & 1.74 (0.78 to 2.09$)$ & $0.55 \pm 0.4$ & 0.17 \\
\hline EWET & 2.07 (0.42 to 0.69$)$ & $0.73 \pm 0.33$ & 0.02 \\
\hline \multicolumn{4}{|c|}{ Adjusted model ${ }^{a}$} \\
\hline EWNT & 2.85 (0.77 to 0.86$)$ & $1.04 \pm 0.52$ & 0.04 \\
\hline NWET & 5.85 (0.56 to 0.72$)$ & $1.76 \pm 0.85$ & 0.03 \\
\hline EWET & 3.13 (0.86 to 0.98 ) & $1.14 \pm 0.6$ & 0.05 \\
\hline
\end{tabular}

Bold values indicate the significant range as a tabular foot

Normal waist normal triglyceride (NWNT) Consider as reference

DII dietary inflammatory index, $O R$ odds ratio, $C l$ confidence interval, $S E$ standard error, EWNT enlarged waist normal triglyceride, NWET normal waist enlarged triglyceride, EWET enlarged waist enlarged

a Adjusted for energy intake, age, plasma insulin levels, marriage status, educational status, economic status and familial obesity history, P value $\leq 0.05$ is significant

HTGWCP. Our findings suggested that, increasing DII scores or consuming pro-inflammatory foods can increase the odds of abnormal phenotypes including; EWNT, NWET, EWET phenotypes compared to NWNT phenotype. According to the results of this study; there was a significant correlation between DII and TG (negatively) so in this study increased DII did not increase TG. However, there was a positive significant relationship between abnormal phenotypes and variables such as insulin, HOMA-IR, TG, weight, height, BMI, SMM, FFM, WHR and WC.

Previous studies have shown that obesity incidence and high WC can be a consequence of previous chronic low-grade inflammation. Therefore, there is a relationship between obesity, especially abdominal obesity and inflammation [35]. Some studies acknowledge that abnormal lipid metabolism plays an important role in the inflammatory response and prognosis of the conditions like obesity and hypertriglyceridemia $[19,36]$. In a cohort study, it was found that the HTGWCP had a prevalence similar to MetS among participants and was associated with the low-grade inflammation seen in obese individuals [37]. Many studies have been shown that DII was correlated with anthropometric components such as weight and WC, and by increasing this score, these components have also increased. A study has shown that the pro-inflammatory diet had a stronger association with WC than with other anthropometric components, among women and men [17, 18]. Many studies in Western societies suggest that the inflammatory properties of diet are positively associated with predictors of CVD, such as increased plasma TG levels and decreased HDL concentrations. Neufcourt et al. showed in a large $(n=3726)$ cohort of French adults that after a follow up of 13 years, the DII score was significantly associated with higher TG and lower HDL levels [14, 15, 38]. One investigation concluded that among police officers, higher DII scores were associated with elevated hs-CRP values and the components of MetS [39].

Our study may be considered as an effective way to prevent the development of obesity. We also evaluated the relationship between some body composition indicators and biochemical parameters and DII, as well as HTGWCP. We selected our target group as women as one of the most sensitive groups in society. We tried to select and adjust some confounders socio-economically because of the association between hypertriglyceridemia and abdominal obesity and such factors.

\section{Conclusion}

It was shown that an increase in DII scores or higher consumption of pro-inflammatory foods can increase all three abnormal phenotypes (EWNT, NWET, EWET) so it may lead to an increase in WC as an important indicator in obesity studies and TG concentration among overweight and obese women. Based on the study, it is recommended that this relationship be done in other target groups such as male adolescent children, etc. It is also better to find more causal clinical studies in this field to find a causal relationship. In addition, researchers can communicate examine many dietary patterns with these phenotypes in their studies.

\section{Limitation}

Our study also had its limitations. Briefly, since the study was a cross-sectional study, causal relationship was not investigated, only correlation. We calculated the DII using FFQ that is recognized to have overreporting and under-reporting bias.

\footnotetext{
Abbreviations

DIl: Dietary inflammatory index; WC: Waist circumference; HTGWCP: Hypertriglyceridemic waist circumference phenotype; TG: Triglyceride; EWNT: Enlarged waist normal TG; NWET: Normal waist enlarged TG; EWET: Enlarged waist enlarged TG; NWNT: Normal waist normal TG; WHO: World Health Organization; CVD: Cardiovascular disease; MeDiet: Mediterranean diet; BMI: Body mass index; WHtR: Waist-to-height ratio; MetSyn: Metabolic syndrome; HDL: Highdensity lipoprotein; FFM: Fat free mass; TNFa: Tumor necrosis factor alpha; hsCRP: High-sensitivity C-reactive protein; IL-1 $\beta$ : Interleukin 1 beta; IL-6: Interleukin 6; IL-10: Interleukin 10; HC: Hip circumference; SMM: Skeletal muscle mass; WHR: Waist hip ratio; ELISA: Enzyme-linked immunosorbent assay; T-chole: Total cholesterol; LDL: Low-density lipoprotein; SGPT: Serum glutamic-pyruvic transaminase; SGOT: Serum glutamic-oxaloacetic transaminase; HOMA-IR: Homeostatic model assessment for insulin resistance; FFQ: Food frequency questionnaire; ANOVA: Analysis of variance; ANCOVA: Analysis of covariance; SD: Standard deviation.
} 


\section{Supplementary Information}

The online version contains supplementary material available at https://doi. org/10.1186/s13104-021-05712-7.

Additional file 1: Table S1. Description of characteristics among tertiles of DII.

Additional file 2: Table S2. Description of characteristics among types of hypertrigeliceridemic waste phenotype.

Additional file 3: Table S3. Crude model and adjusted model for Relationship between hyper triglyceridemic waist circumference phenotype and DII.

\section{Acknowledgements}

This study was supported by Tehran University of Medical Sciences Grant (ID: 98-3-212-46728). We thank all the participants in this study, as well as the school of Nutritional and Dietetics at Tehran University of medical sciences.

\section{Authors' contributions}

AT, AM, HM contributed to conception and design. AT, AM and SM Contributed to all experimental work. AM, Contributed to data and statistical analysis. KM and S-AK supervised the whole project. All authors performed editing and approving the final version of this paper for submission, also participated in the finalization of the manuscript. All authors read and approved the final manuscript.

\section{Funding}

This study was supported by Tehran University of Medical Sciences (Grant No: 98-3-212-46728).

\section{Availability of data and materials}

The data that support the findings of this study are available from Dr. Khadijeh Mirzaei but restrictions apply to the availability of these data, which were used under license for the current study, and so are not publicly available. Data are however available from the authors upon reasonable request and with permission of Dr. Khadijeh Mirzaei.

\section{Declarations}

\section{Ethics approval and consent to participate}

The study protocol was approved by the ethics committee of Tehran University of medical sciences (IR.TUMS.MEDICINE.REC.1399.636) and is acknowledged by authors. All participants signed a written informed consent.

\section{Consent for publication}

Not applicable.

\section{Competing interests}

The authors declare that there is no conflict of interest in this study.

\section{Author details}

${ }^{1}$ Department of Community Nutrition, School of Nutritional Sciences and Dietetics, Tehran University of Medical Sciences (TUMS), P.O. Box: 14155-6117, Tehran, Iran. ${ }^{2}$ Department of Clinical Nutrition, School of Nutritional Sciences and Dietetics, Tehran University of Medical Sciences, Tehran, Iran.

\section{Received: 1 April 2021 Accepted: 23 July 2021}

Published online: 16 August 2021

\section{References}

1. Ng M, Fleming T, Robinson $M$, et al. Global, regional, and national prevalence of overweight and obesity in children and adults during 1980-2013: a systematic analysis for the Global Burden of Disease Study 2013. Lancet. 2014;384(9945):766-81.
2. Saeidlou SN, Vahabzadeh D, Babaei F, Vahabzadeh Z. Seasonal variations of vitamin D and its relation to lipid profile in Iranian children and adults. J Health Population Nutr. 2017:36(1):1-7.

3. Heber D. An integrative view of obesity. Am J Clin Nutr. 2010:91(1):280S-283S.

4. Ahluwalia N, Andreeva VA, Kesse-Guyot E, Hercberg S. Dietary patterns, inflammation and the metabolic syndrome. Diabetes Metab. 2013;39(2):99-110.

5. Barbaresko J, Koch M, Schulze MB, Nöthlings U. Dietary pattern analysis and biomarkers of low-grade inflammation: a systematic literature review. Nutr Rev. 2013;71(8):511-27.

6. Calder PC, Ahluwalia N, Brouns F, et al. Dietary factors and lowgrade inflammation in relation to overweight and obesity. $\mathrm{Br} \mathrm{J}$ Nutr. 2011:106(S3):S1-78.

7. Viscogliosi G, Cipriani E, Liguori ML, et al. Mediterranean dietary pattern adherence: associations with prediabetes, metabolic syndrome, and related microinflammation. Metab Syndr Relat Disord. 2013;11(3):210-6.

8. Schwingshackl L, Hoffmann G. Mediterranean dietary pattern, inflammation and endothelial function: a systematic review and meta-analysis of intervention trials. Nutr Metab Cardiovasc Dis. 2014;24(9):929-39.

9. Bulló M, Casas-Agustench P, Amigó-Correig P, Aranceta J, Salas-Salvadó J. Inflammation, obesity and comorbidities: the role of diet. Public Health Nutr. 2007;10(10A):1164-72.

10. Funtikova AN, Benítez-Arciniega AA, Gomez SF, Fitó M, Elosua R, Schröder H. Mediterranean diet impact on changes in abdominal fat and 10-year incidence of abdominal obesity in a Spanish population. Br J Nutr. 2014;111(8):1481-7

11. Esmaillzadeh A, Kimiagar M, Mehrabi Y, Azadbakht L, Hu FB, Willett WC. Dietary patterns and markers of systemic inflammation among Iranian women. J Nutr. 2007;137(4):992-8.

12. Salas-Salvado J, Bullo M, Garcia-Lorda P, et al. Subcutaneous adipose tissue cytokine production is not responsible for the restoration of systemic inflammation markers during weight loss. Int J Obes. 2006;30(12):1714-20.

13. Ruiz-Canela M, Zazpe I, Shivappa N, et al. Dietary inflammatory index and anthropometric measures of obesity in a population sample at high cardiovascular risk from the PREDIMED (PREvencion con Dleta MEDiterranea) trial. Br J Nutr. 2015;113(6):984-95.

14. Camargo-Ramos CM, Correa-Bautista JE, Correa-Rodríguez M, RamírezVélez R. Dietary inflammatory index and cardiometabolic risk parameters in overweight and sedentary subjects. Int J Environ Res Public Health. 2017;14(10):1104

15. Neufcourt L, Assmann K, Fezeu L, et al. Prospective association between the dietary inflammatory index and metabolic syndrome: findings from the SU. VI. MAX study. Nutr Metab Cardiovasc Dis. 2015;25(11):988-96.

16. Blackburn P, Lemieux I, Lamarche B, et al. Hypertriglyceridemic waist: a simple clinical phenotype associated with coronary artery disease in women. Metabolism. 2012;61(1):56-64.

17. Ramallal R, Toledo E, Martínez JA, et al. Inflammatory potential of diet, weight gain, and incidence of overweight/obesity: the SUN cohort. Obesity. 2017:25(6):997-1005.

18. Hermsdorff HHM, Zulet MÁ, Puchau B, Martínez JA. Central adiposity rather than total adiposity measurements are specifically involved in the inflammatory status from healthy young adults. Inflammation. 2011;34(3):161-70

19. Chen S, Guo X, Yu S, et al. Hypertriglyceridemic waist phenotype and metabolic abnormalities in hypertensive adults: a STROBE compliant study. Medicine. 2016;95(49):e5613.

20. Azadbakht L, Esmaillzadeh A. Dietary diversity score is related to obesity and abdominal adiposity among Iranian female youth. Public Health Nutr. 2011;14(1):62-9.

21. Salehi-Abargouei A, Esmaillzadeh A, Azadbakht L, et al. Nutrient patterns and their relation to general and abdominal obesity in Iranian adults: findings from the SEPAHAN study. Eur J Nutr. 2016;55(2):505-18.

22. Ackerson LK, Subramanian S. Domestic violence and chronic malnutrition among women and children in India. Am J Epidemiol. 2008;167(10):1188-96. 
23. Hosseinpanah F, Barzin M, Eskandary PS, Mirmiran P, Azizi F. Trends of obesity and abdominal obesity in Tehranian adults: a cohort study. BMC Public Health. 2009;9(1):1-9.

24. Suara SB, Siassi F, Saaka M, Foroshani AR, Sotoudeh G. Association between Carbohydrate Quality Index and general and abdominal obesity in women: a cross-sectional study from Ghana. BMJ Open. 2019;9(12):e033038.

25. Leddy AM, Roque A, Sheira LA, et al. Food insecurity is associated with inflammation among women living with HIV. J Infect Dis. 2019;219(3):429-36.

26. Holtrop M, Heltshe S, Shabanova $V$, et al. A prospective study of the effects of sex hormones on lung function and inflammation in women with cystic fibrosis. Ann Am Thorac Soc. 2021;18:1158-66.

27. Vahid F, Shivappa N, Hekmatdoost A, Hebert JR, Davoodi SH, Sadeghi M. Association between Maternal Dietary Inflammatory Index (DII) and abortion in Iranian women and validation of DII with serum concentration of inflammatory factors: case-control study. Appl Physiol Nutr Metab. 2017:42(5):511-6.

28. Shivappa N, Sandin S, Löf M, Hébert JR, Adami H-O, Weiderpass E. Prospective study of dietary inflammatory index and risk of breast cancer in Swedish women. Br J Cancer. 2015;113(7):1099-103.

29. Ghodoosi N, Mirzababaei A, Rashidbeygi E, et al. Associations of dietary inflammatory index, serum levels of MCP-1 and body composition in Iranian overweight and obese women: a cross-sectional study. BMC Res Notes. 2020;13(1):1-7.

30. Laouali N, Mancini FR, Hajji-Louati M, et al. Dietary inflammatory index and type 2 diabetes risk in a prospective cohort of 70,991 women followed for 20 years: the mediating role of BMI. Diabetologia. 2019;62(12):2222-32

31. Mirmiran P, Esfahani FH, Mehrabi Y, Hedayati M, Azizi F. Reliability and relative validity of an FFQ for nutrients in the Tehran lipid and glucose study. Public Health Nutr. 2010;13(5):654-62.
32. Shivappa N, Steck SE, Hurley TG, Hussey JR, Hébert JR. Designing and developing a literature-derived, population-based dietary inflammatory index. Public Health Nutr. 2014;17(8):1689-96.

33. Esfahani FH, Asghari G, Mirmiran P, Azizi F. Reproducibility and relative validity of food group intake in a food frequency questionnaire developed for the Tehran Lipid and Glucose Study. J Epidemiol. 2010;20(2):150-8.

34. Amini M, Esmaillzadeh A, Sadeghi M, Mehvarifar N, Amini M, Zare M. The association of hypertriglyceridemic waist phenotype with type 2 diabetes mellitus among individuals with first relative history of diabetes. J Res Med Sci. 2011;16(2):156.

35. Richard C, Couture P, Desroches S, Lamarche B. Effect of the Mediterranean diet with and without weight loss on markers of inflammation in men with metabolic syndrome. Obesity. 2013;21(1):51-7.

36. Neeland IJ, Ayers CR, Rohatgi AK, et al. Associations of visceral and abdominal subcutaneous adipose tissue with markers of cardiac and metabolic risk in obese adults. Obesity. 2013;21(9):E439-47.

37. Rogowski O, Shapira I, Steinvil A, Berliner S. Low-grade inflammation in individuals with the hypertriglyceridemic waist phenotype: another feature of the atherogenic dysmetabolism. Metabolism. 2009;58(5):661-7.

38. Vissers LE, Waller M, van der Schouw YT, et al. A pro-inflammatory diet is associated with increased risk of developing hypertension among middle-aged women. Nutr Metab Cardiovasc Dis. 2017;27(6):564-70.

39. Wirth M, Burch J, Shivappa N, et al. Association of a dietary inflammatory index with inflammatory indices and the metabolic syndrome among police officers. J Occup Environ Med Am Coll Occup Environ Med. 2014;56(9):986.

\section{Publisher's Note}

Springer Nature remains neutral with regard to jurisdictional claims in published maps and institutional affiliations.
Ready to submit your research? Choose BMC and benefit from:

- fast, convenient online submission

- thorough peer review by experienced researchers in your field

- rapid publication on acceptance

- support for research data, including large and complex data types

- gold Open Access which fosters wider collaboration and increased citations

- maximum visibility for your research: over $100 \mathrm{M}$ website views per year

At BMC, research is always in progress.

Learn more biomedcentral.com/submissions 